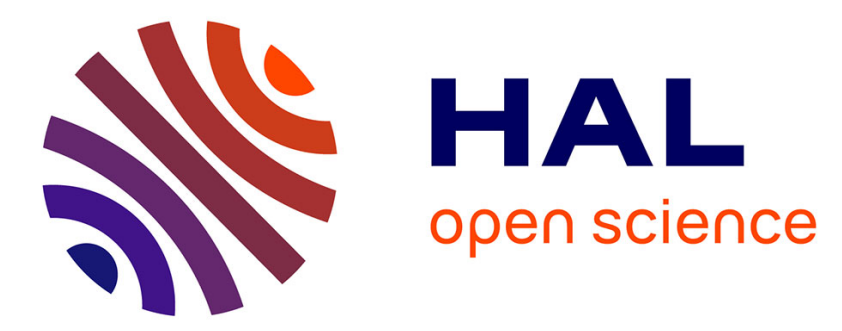

\title{
How much do solar cycle variations impact long term effect predictions at LEO?
}

\author{
Sébastien Bourdarie, Philippe Calvel, Catherine Barillot, Laurent Rey, \\ Tommaso Parrinello, Berta Hoyos, Robert Ecoffet
}

\section{To cite this version:}

Sébastien Bourdarie, Philippe Calvel, Catherine Barillot, Laurent Rey, Tommaso Parrinello, et al.. How much do solar cycle variations impact long term effect predictions at LEO?. IEEE Transactions on Nuclear Science, 2020, 67 (10), pp.2196-2202. 10.1109/TNS.2020.3008251 . hal-02505636v2

\section{HAL Id: hal-02505636 \\ https://hal.science/hal-02505636v2}

Submitted on 1 Mar 2021

HAL is a multi-disciplinary open access archive for the deposit and dissemination of scientific research documents, whether they are published or not. The documents may come from teaching and research institutions in France or abroad, or from public or private research centers.
L'archive ouverte pluridisciplinaire HAL, est destinée au dépôt et à la diffusion de documents scientifiques de niveau recherche, publiés ou non, émanant des établissements d'enseignement et de recherche français ou étrangers, des laboratoires publics ou privés. 


\title{
How Much Do Solar Cycle Variations Impact Long-Term Effect Predictions at LEO?
}

\author{
S. Bourdarie ${ }^{\circledR}$, P. Calvel, C. Barillot, L. Rey, T. Parrinello, B. Hoyos, and R. Ecoffet, Member, IEEE
}

\begin{abstract}
An eight-year long flight database from an error detection and correction (EDAC) counter implemented onboard an altimeter flying on CryoSat-2 spacecraft at $715-\mathrm{km}$ altitude is analyzed to investigate the impact of solar cycle variations on long-term effect predictions. In situ observations are then compared to various specification models including legacy models as well as models under developments.
\end{abstract}

Index Terms - Radiation belts, space radiation models.

\section{INTRODUCTION}

$\mathbf{T}$ O DESIGN space missions, the current standard models, AP8 [1] and AE8 [2], are widely used in space industries. These models were developed by National Aeronautics and Space Administration (NASA) at the end of the 1970s and beginning of the 1980s. Two versions of each model were produced (AP8 max and AP8 min, AE8 max and AE8 min) to reflect solar cycle variations only at $L<3$ and altitudes less than $1000 \mathrm{~km}$. ECSS-E10-04 standard recommends the use of AP8 min plus AE8 max to design space vehicles. This is considered to be a conservative approach because AP8 min fluxes are higher than AP8 max ones and AE8 max fluxes are higher than AE8 min ones. Recently, two initiatives to upgrade these models are on-going: AE9/AP9 [3] and GREEN [4] specification models. Currently, solar cycle variations are not directly accounted for in AE9/AP9 model, while they are in GREEN. Note that in AE9/AP9, solar cycle variations are indirectly accounted for by the statistics provided in the perturbed mean and Monte-Carlo AE9/AP9 models knowing that they do not indicate when the peak flux will be reached.

According to the variety of space environment models now available with or without solar cycle variations, it is important to address the following question to help space industries in their future development: how much do solar cycle variations impact long-term effect (average single event effect (SEE) rate, ionizing dose, and displacement damage) predictions?

S. Bourdarie is with the ONERA-The French Aerospace Lab/ Département Environnement Spatial, 31400 Toulouse, France (e-mail: sebastien.bourdarie@onera.fr).

P. Calvel, C. Barillot, and L. Rey are with Thales Alenia Space, 31027 Toulouse, France.

T. Parrinello and B. Hoyos are with the ESA/ESRIN, 100044 Frascati, Italy. R. Ecoffet is with the CNES, 31401 Toulouse, France.

Color versions of one or more of the figures in this article are available online at http://ieeexplore.iee.org.
This article addresses this issue for trapped protons at Low Earth Orbit (LEO). To do so, flight data from error detection and correction (EDAC) counter collected over several years at LEO will be used. Then predictions obtained with AP8 min and max, AP9 Mean, and GREEN-p will be compared to flight data to deduce the most appropriate model to be used at LEO.

In Section II, flight data are presented as well as support data necessary to perform effect calculations, in Section III, model predictions will be given, and in Section IV, results will be discussed.

\section{Flight Data}

CryoSat-2 is a European Space Agency (ESA) satellite flying on an LEO (near circular orbit: 715-735 km, inclination: $92^{\circ}, 14.52$ revolutions/day). It was launched on April 8, 2010. The evolution of perigee, apogee, and inclination from launch date to April 4, 2019, is given in Fig. 1. One can see that the orbit is almost the same over this time range, that is, the spacecraft will encounter the same environment along the mission life time.

In [5], single-event phenomena (bit flips) recorded by an EDAC onboard the SAR Interferometer Radar Altimeter (SIRAL) being built by Thales Alenia Space (TAS) were analyzed. These errors are automatically corrected, listed by the EDAC counter, and downloaded on ground station every month. The memory map of this payload is made of 10 static random access memory (SRAM) (1-MB SRAM M65608, 0.5- $\mu \mathrm{m}$ CMOS process, developed by ATMEL in cooperation with the ESA).

The total number of single-event upset (SEU) recorded by the EDAC from November 1, 2010, to March 31, 2019, is 6927. The longitude-latitude event distribution is given in Fig. 2. The number of events is sufficient to nicely image the South Atlantic Anomaly (SAA). Following [5], SEU events can be sorted out to identify those induced by trapped protons in the radiation belts (McIlwain $L<1.9$ ) to those induced by galactic cosmic rays (GCRs) or solar energetic particles (SEPs) (McIlwain $L>1.9$ ). As already reported in [5], most SEUs are recorded in the SAA (87.3\%), while the SEUs at high and low latitudes can be attributed to GCRs and SEPs $(12.7 \%)$. Note that since the time coverage is longer here than in [5], the percentages obtained here are slightly different $(\sim 1 \%)$. The mean SEU rates then obtained are 1.88 and $0.28 \mathrm{SEU} /$ day attributed to trapped protons and GCRs plus SEPs, respectively, over the full time period.

The time evolution of cumulated SEUs as well as the year average daily SEU rates attributed to trapped protons and 


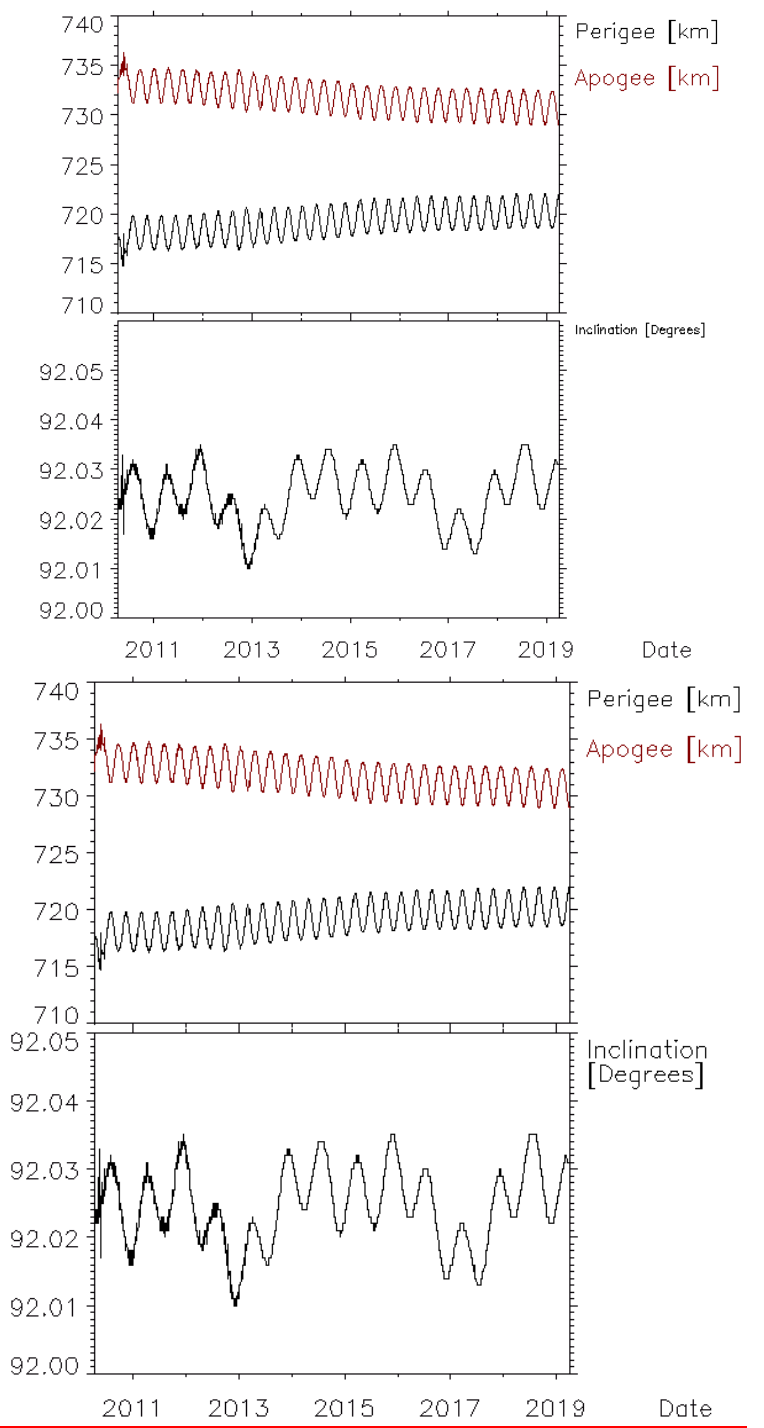

Fig. 1. CryoSat-2 orbital parameters out of two line elements versus time.

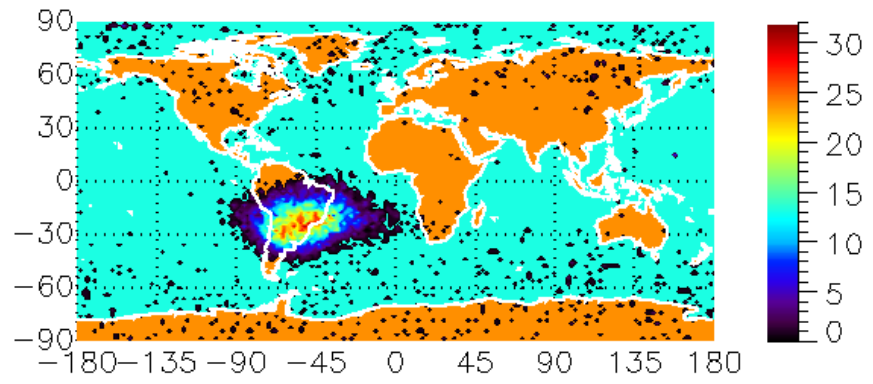

Fig. 2. SEU recorded by the EDAC implemented on the CryoSat-2/SIRAL payload from November 1, 2010, to March 31, 2019.

GCRs plus SEP are plotted versus time in Fig. 3. The cumulated SEU values (top two panels) confirm that events are dominated by the trapped proton contribution. From the cumulative SEU values, it is then possible to compute year average daily SEU rates attributed to trapped protons (third panel from top) and GCR plus SEP (fourth panel from top). In both cases, the solar cycle modulation in SEU rates can be clearly seen. Note that such variations cannot be attributed to an orbit change as apogee and perigee only evolve by $5 \mathrm{~km}$ from 2011 to

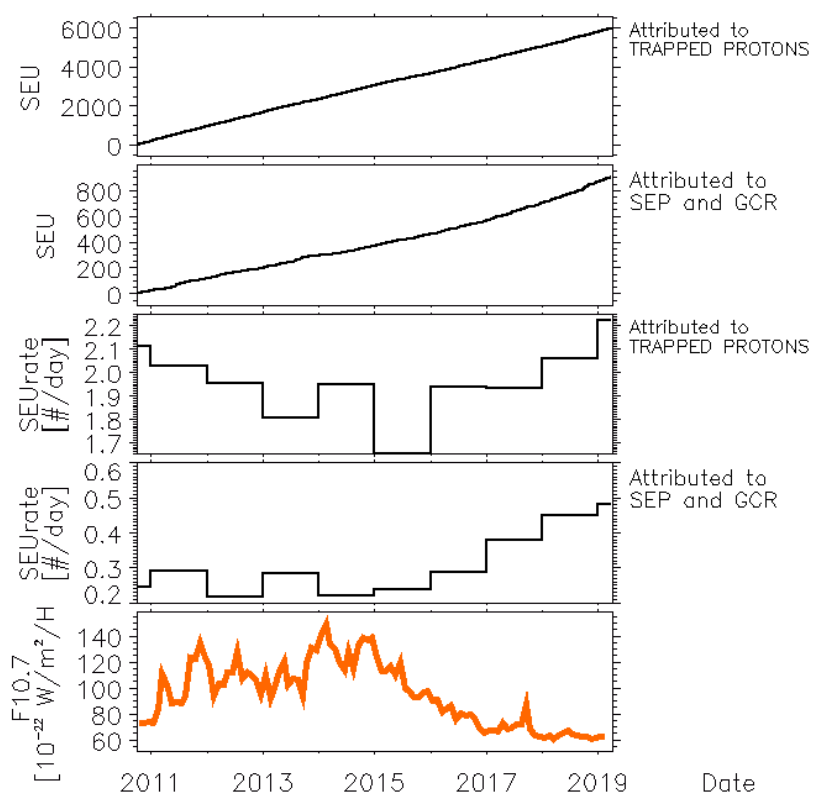

Fig. 3. (Top two panels) Cumulated SEUs as well as the (second and third panels from bottom) SEU rates attributed to trapped protons and GCRs plus SEP versus time. The F10.7 solar radio flux is given to illustrate the solar cycle evolution.

2019 and the inclination remains unchanged. As expected, the SEU rate attributed to GCR plus SEP is the lowest at solar cycle maximum phase (April 2014) and is increasing by a factor of 2 from solar maximum (0.22 SEU/day in 2014) to almost solar minimum (0.48 SEU/day in 2019). The SEU rate attributed to trapped protons is decreasing slowly in time from year 2011 (2.1 SEU/day) to year 2015 (1.65 SEU/day), that is, all along solar maximum phase a $30 \%$ decrease is found. Then from year 2015 (1.65 SEU/day) to year 2019 (2.22 SEU/day), a slow increase in SEU daily rates is observed, that is, all along declining phase a $30 \%$ increase is found.

During SEP events, January 27-29, 2012, and March 7-11, 2012, a net increase (two events can be attributed to each SEP event) is found in cumulated SEU values attributed to GCR plus SEP, while no significant changes are visible in cumulated SEU values attributed to trapped protons (Fig. 4).

To find out how accurate trapped proton specification models are cumulated as well as SEU daily rates will be evaluated is explained in Section III. To calculate an accurate SEU rate of mass memory of the SIRAL payload, the 3-D shielding around the SRAM device and the device cross section versus proton energy must be well known.

The distribution of shielding thicknesses seen by the SRAM device onboard CryoSat-2/SIRAL was calculated by a sector analysis carried out by TAS Company using the FASTRAD software [6]. The solid angle viewed from SRAM M65608 is decomposed into 1800 sectors of equal value, that is, 30 steps in polar angles and 60 steps in azimuthal angles (see [5] for more details). The 3-D CAD model of the spacecraft is then simplified to the distribution of shielding in the $4 \pi$ steradian obtained with FASTRAD.

The M65608 SRAM has been tested under heavy ions and proton beams by ESA. In particular, three test campaigns under proton beam were performed and are denoted by 


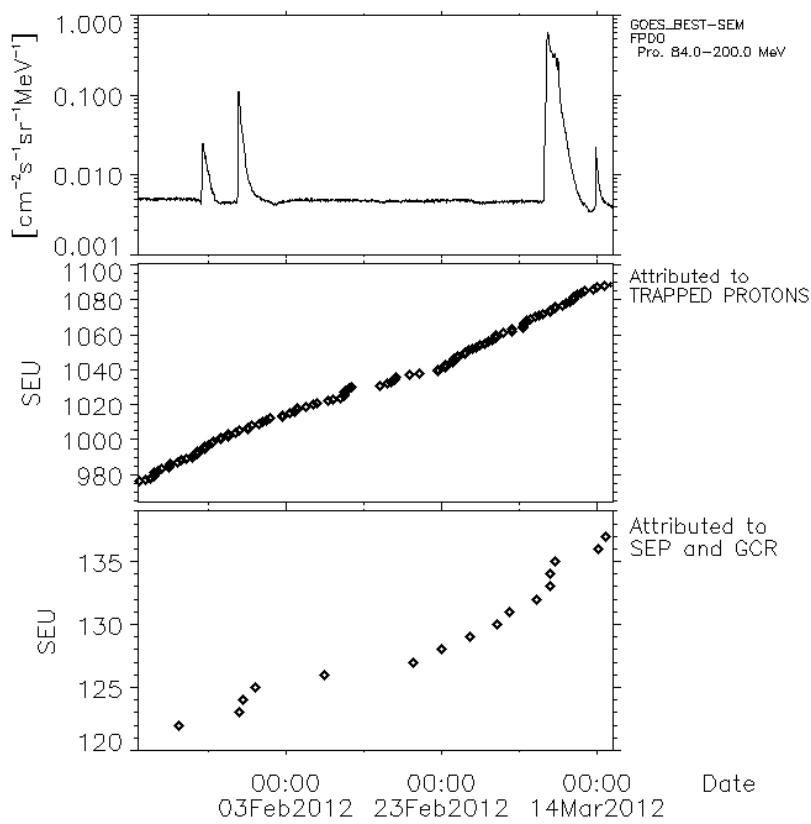

Fig. 4. (Bottom two panels) Cumulated SEUs attributed to trapped protons and GCRs plus SEP from January 15, 2012, to March 15, 2012, and SEPs measured by Geostationary Operational Environmental Satellite (GOES) spacecraft.

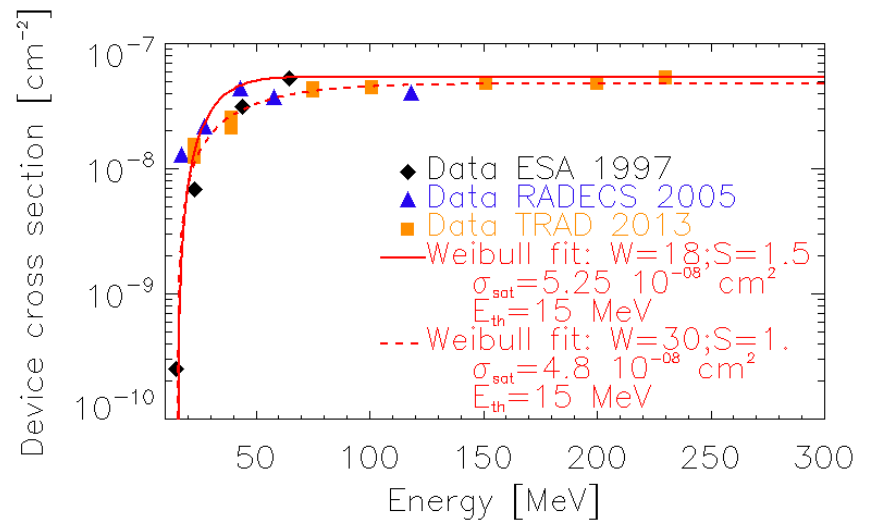

Fig. 5. M65608 SRAM device cross section versus proton energy. Symbols indicate results from test campaigns, while the solid and dotted lines represent a high and a low Weibull fit, respectively.

"ESA 1997" [7], "RADECS 2005" [8], and "TRAD 2013" [9]. The SEU cross section versus proton energy is given in Fig. 5 and can be fitted by a Weibull fit function. Two fits can be defined, one "high" which is pessimistic for prediction (the solid line in Fig. 5) and one "low" which is optimistic for prediction (the dashed line in Fig. 5), with the following parameters: $W=18, s=1.5$, an energy threshold of $15 \mathrm{MeV}$ and a saturated cross section of $5.28 \cdot 10^{-8} \mathrm{~cm}^{2} /$ device and $W=30$, $s=1$, an energy threshold of $15 \mathrm{MeV}$ and a saturated cross section of $4.8 \cdot 10^{-8} \mathrm{~cm}^{2} /$ device can be found, respectively.

To compute SEU cumulated values out of environment specification models, the same strategy is applied here as in [5].

\section{SPecification Model Predictions}

Although AP8 has been widely used to design spacecraft for several decades, alternatives like AP9 and GREEN-p are now
TABLE I

Main Characteristics of Trapped Proton Specification Models

\begin{tabular}{|l|l|l|l|}
\hline & AP8 & AP9 & GREEN-p \\
\hline Spatial coverage & $1.15<\mathrm{L}<6.6$ & $0.98<\mathrm{L}<12.4$ & $0.98<\mathrm{L}<6.6$ \\
& All latitudes & All latitudes & All latitudes \\
\hline $\begin{array}{l}\text { Energy } \\
\text { coverage }\end{array}$ & $\begin{array}{l}100 \mathrm{keV}-300 \\
\mathrm{MeV}\end{array}$ & $\begin{array}{l}100 \mathrm{keV}-2 \\
\mathrm{GeV}\end{array}$ & $\begin{array}{l}100 \mathrm{keV}-800 \\
\mathrm{MeV}\end{array}$ \\
\hline $\begin{array}{l}\text { Magnetic field } \\
\text { model }\end{array}$ & $\begin{array}{l}\text { Fixed to epoch } \\
1970 \text { or 1960 }\end{array}$ & Yearly & Yearly \\
\hline $\begin{array}{l}\text { Solar cycle } \\
\text { variations }\end{array}$ & $\begin{array}{l}\text { Two states: } \\
\text { solar min or } \\
\text { solar max }\end{array}$ & None & Yearly \\
\hline Data sets epoch & $1958-1970$ & $1969-2016$ & $1979-2018$ \\
\hline
\end{tabular}

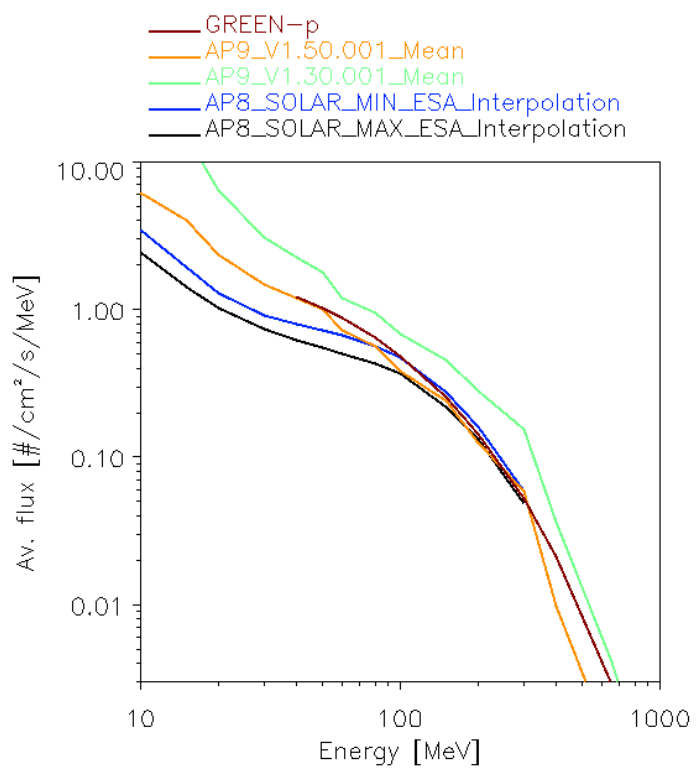

Fig. 6. Differential proton flux at CryoSat-2 deduced from AP8 min, AP9 Mean V1.30.001, AP9 Mean V1.50.001, and GREEN-p models.

available. All three models offer a global spatial and energy coverage of the radiation belts. Their main characteristics are summarized in Table I.

To compute the trapped proton environment encountered by CryoSat-2 spacecraft, its orbit parameters (altitude, latitude, and longitude) are computed with a 20-s time resolution from two line elements [10] using SGP4 orbit propagator [11] from launch date to April 1, 2019. Then the proton fluxes are estimated at all orbital locations from AP8 min with ESA interpolations, AP8 max with ESA interpolations, AP9 V1.30.001 Mean, AP9 V1.50.001 Mean, and GREEN-p. All calculations are performed using the Benchmark of Ionizing Space Environment tool [5].

A comparison of the mission average differential trapped proton spectrum (assumed to be isotropic at the spacecraft) and transmitted (at the chip level) deduced from AP8 min with ESA interpolation, AP8 max with ESA interpolation, AP9 Mean V1.30.001, AP9 Mean V1.50.001, and GREEN-p models are given in Figs. 6 and 7, respectively. Note that the transmitted proton fluxes at the chip level are calculated from a MCNPx V2.7.0 Monte-Carlo run accounting for the 3-D distribution of shielding surrounding the chip. For protons with energy greater than $40 \mathrm{MeV}$ at the spacecraft or greater than $10 \mathrm{MeV}$ at the chip, AP9 Mean V1.30.001 fluxes are the 


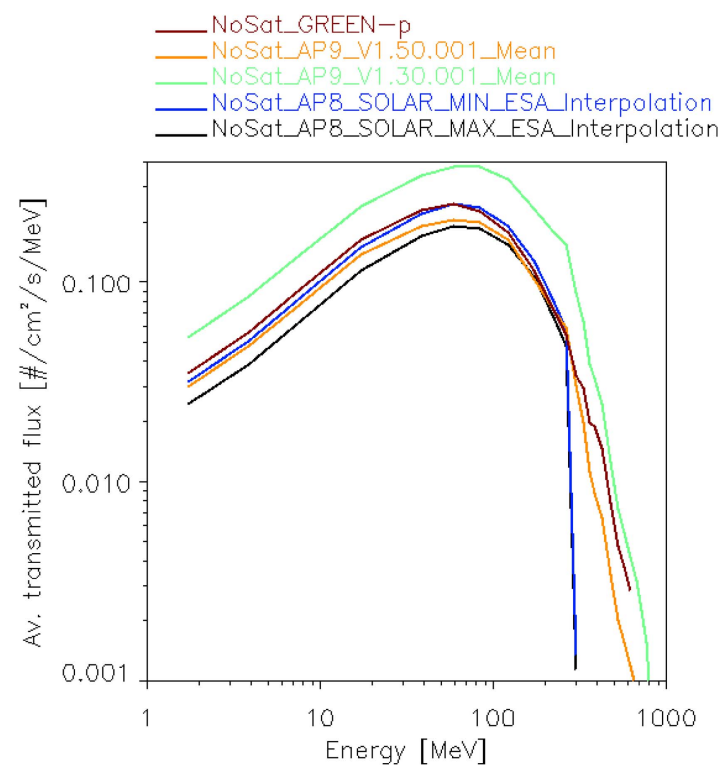

Fig. 7. Transmitted differential proton flux at the CryoSat-2/SIRAL/ chip level deduced from AP8 min, AP9 Mean V1.30.001, AP9 Mean V1.50.001, and GREEN-p models.

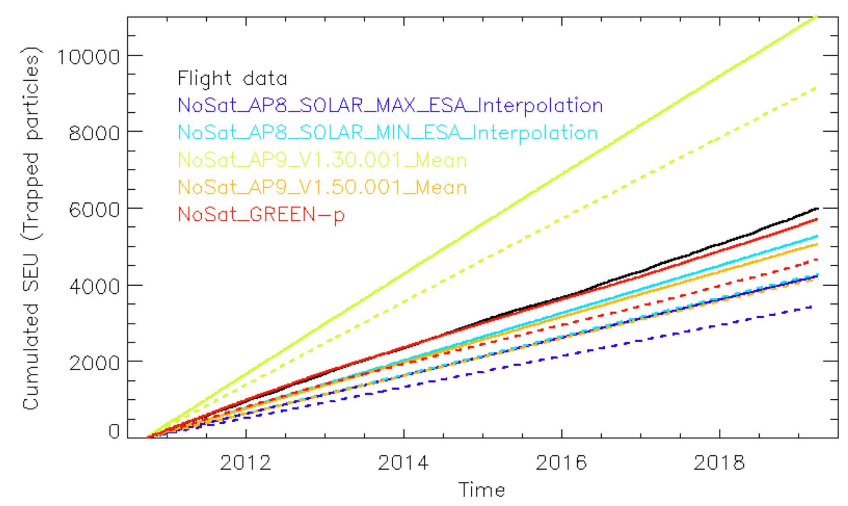

Fig. 8. Comparison of the cumulated SEUs measured by the EDAC onboard CryoSat-2/SIRAL and predicted from AP8 min with ESA interpolation, AP8 max with ESA interpolation, AP9 Mean V1.30.001, AP9 Mean V1.50.001, and GREEN-p models. Solid lines are obtained when the Weibull fit "high" is used, while the dashed lines are obtained when the Weibull fit "low" is considered.

highest ones, while AP8 max fluxes are the lowest ones, the differences being close to a factor 2 .

A comparison of cumulated SEUs predicted from AP8 min with ESA interpolation, AP8 max with ESA interpolation, AP9 Mean V1.30.001, AP9 Mean V1.50.001, and GREEN-p is shown in Fig. 8. At the end of the time period under study, it is found that

- AP8 min with ESA interpolation underestimates the cumulated SEUs by $12.2 \%-28.6 \%$ according to the Weibull fit "high" or "low" being considered, respectively;

- AP8 max with ESA interpolation underestimates the cumulated SEUs by $29.5 \%-42.5 \%$ according to the Weibull fit "high" or "low" being considered, respectively;

- AP9 Mean V1.50.001 underestimates the cumulated SEUs by $15.6 \%-30.7 \%$ according to the Weibull fit "high" or "low" being considered, respectively;

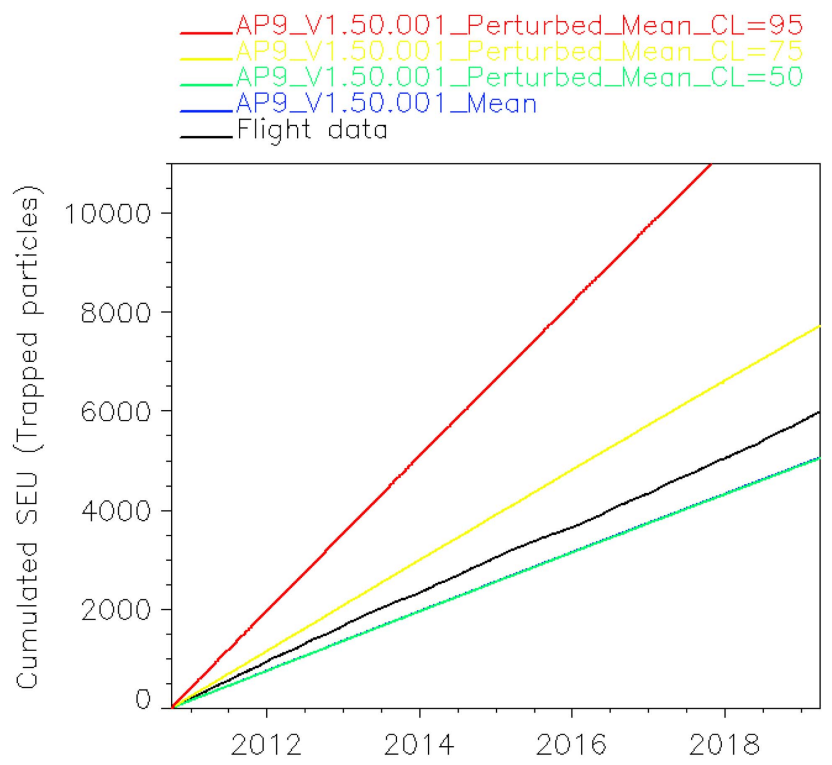

Fig. 9. Comparison of the cumulated SEUs measured by the EDAC onboard CryoSat-2/SIRAL and predicted from AP9 Perturbed Mean V1.50.001 median $(\mathrm{CL}=50)$, 75th percentile $(\mathrm{CL}=75)$, and 95th percentile $(\mathrm{CL}=95)$. The Weibull fit "high" is used.

- GREEN-p underestimates the cumulated SEUs by 4.7\%-22.4\% according to the Weibull fit "high" or "low" being considered, respectively;

- AP9 Mean V1.30.001 overestimates the cumulated SEUs by $83.9 \%-52.7 \%$ according to the Weibull fit "high" or "low" being considered, respectively.

AP9 models offer other options to investigate the long-term space environment. In particular, the "perturbed mean" and "Monte-Carlo" modes allow defining the trapped particle flux for a given confidence level (CL). In this prospect, the AP9 V1.50.001 "perturbed mean" model has been used following the same strategy as for the other engineering models. The median, 75th percentile, and 95th percentile proton fluxes were computed out of 40 scenarios. Then the cumulated SEU events have been deduced using the Weibull fit "high" (Fig. 9). The following has been found.

- AP9 V1.50.001 "Perturbed Mean" median provides exactly the same result as the one deduced from AP9 V1.50.001 Mean model. It underestimates the cumulated SEUs by $15.6 \%$.

- AP9 V1.50.001 "Perturbed Mean" 75th percentile overestimates the cumulated SEUs by $28.9 \%$.

- AP9 V1.50.001 “Perturbed Mean" 95th percentile overestimates the cumulated SEUs by $119.5 \%$.

- Flight data correspond to an CL comprises between 50 and 75 according to AP9 "Perturbed Mean" V1.50.001 model.

To analyze which energy range of the input transported spectrum at the cell level is the most relevant to predict the total SEU number in the SRAM, the cumulative percentage of total SEU is plotted versus energy in Fig. 10. Fifty percent of events are attributed to protons with energy greater than 112-150 MeV depending on the specification model and the Weibull fit being considered. Full details are provided 


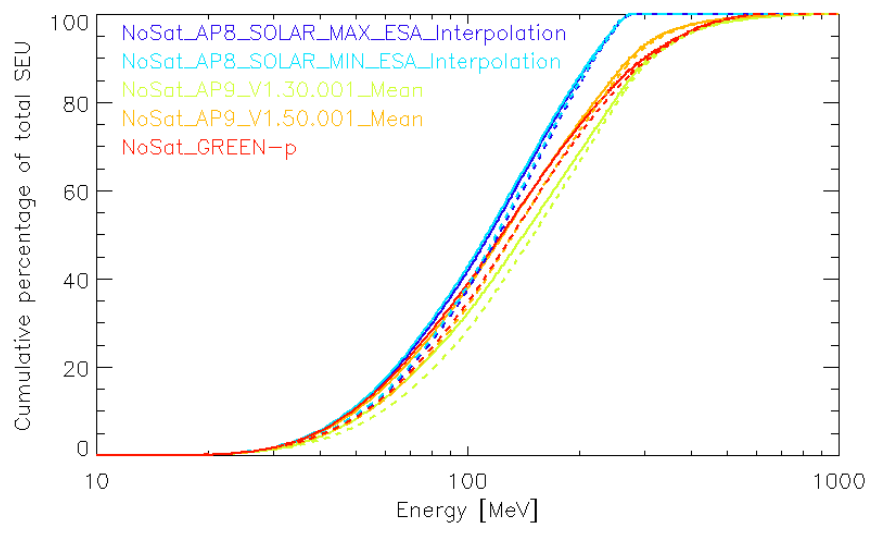

Fig. 10. Cumulative percentage of total SEU versus energy predicted from specification models. Solid lines are obtained when the Weibull fit "high" is used, while the dashed lines are obtained when the Weibull fit "low" is considered.

TABLE II

Energy [MeV] AT Which 50\% of Cumulated Events Is Predicted VERSUS THE SPECIFICATION AND THE WEIBULL FIT CONSIDERED

\begin{tabular}{|l|l|l|}
\hline & \multicolumn{3}{|l|}{$\begin{array}{l}\text { Energy }[\mathrm{MeV}] \text { at which 50\% of } \\
\text { cumulated events are predicted }\end{array}$} \\
\hline & $\begin{array}{l}\text { Weibull fit } \\
\text { "high" }\end{array}$ & $\begin{array}{l}\text { Weibull } \\
\text { "low" fit }\end{array}$ \\
\hline AP8 solar max & 114. & 121. \\
\hline AP8 solar min & 112. & 119. \\
\hline AP9 Mean V1.30.001 & 141. & 150. \\
\hline AP9 Mean V1.50.001 & 124. & 132. \\
\hline GREEN-p & 123. & 132. \\
\hline
\end{tabular}

in Table II. Note that in all cases, $50 \%$ of events are attributed to protons with energies corresponding to the saturated device cross section.

While AP8 solar min and solar max provide proton spectrum up to $300 \mathrm{MeV}$ (i.e., $276 \mathrm{MeV}$ at the sensitive cell), AP9 Mean V1.30.001, AP9 Mean V1.50.001, and GREEN-p indicate that $14.2 \%, 9.57 \%$, and $12.3 \%$ of events are attributed to protons with incident energy greater than $300 \mathrm{MeV}$. It is also found that $99 \%$ of events are predicted if the spectrum is truncated at $500 \mathrm{MeV}$ from AP9 Mean models and GREEN-p and for both Weibull fits. This is clearly indicating that a proton specification model with a spectrum defined up to $500 \mathrm{MeV}$ is sufficient in the present case study.

To investigate which model reflects best the solar cycle modulation, daily SEU rates (averaged over each calendar year) can be deduced from those predictions. Results are given in Fig. 11 for each specification model. The solid lines are deduced when the Weibull fit "high" is considered, that is, it provides upper predicted values because the device cross section is pessimistic in this case and the dashed lines are computed using the Weibull fit "low," that is, it leads to lower predicted values because the device cross section is optimistic in this case. By definition, AP8 min and max do not vary along the solar cycle. Although there is an offset down in AP8 predictions, the amplitude between AP8 min and AP8 max is quite representative of what to expect between solar max and solar minimum phase. While AP9 V1.30.001 Mean and AP9 V1.50.001 do not include solar cycle variations, results

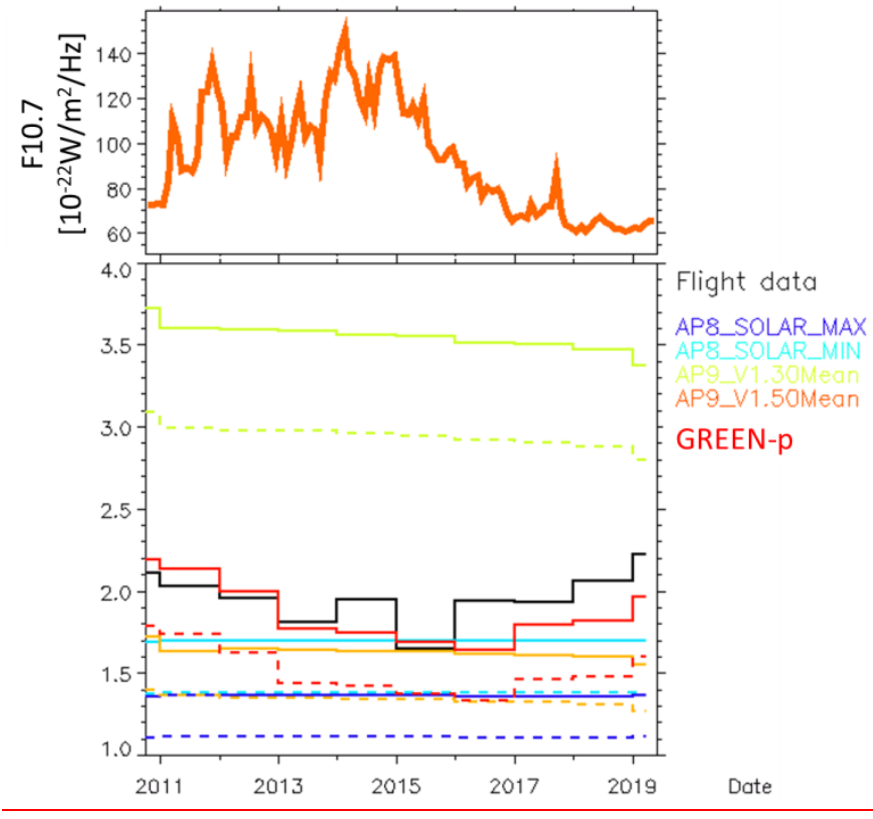

Fig. 11. (Bottom) Daily SEU rates from in-flight data and predicted with AP8 min with ESA interpolation, AP8 max with ESA interpolation, AP9 Mean V1.30.001, AP9 Mean V1.50.001, and GREEN-p. Solid lines are obtained when the Weibull fit "high" is used, while the dashed lines are obtained when the Weibull fit "low" is considered. (Top) Solar radio flux at $10.7 \mathrm{~cm}, \mathrm{~F} 10.7$.
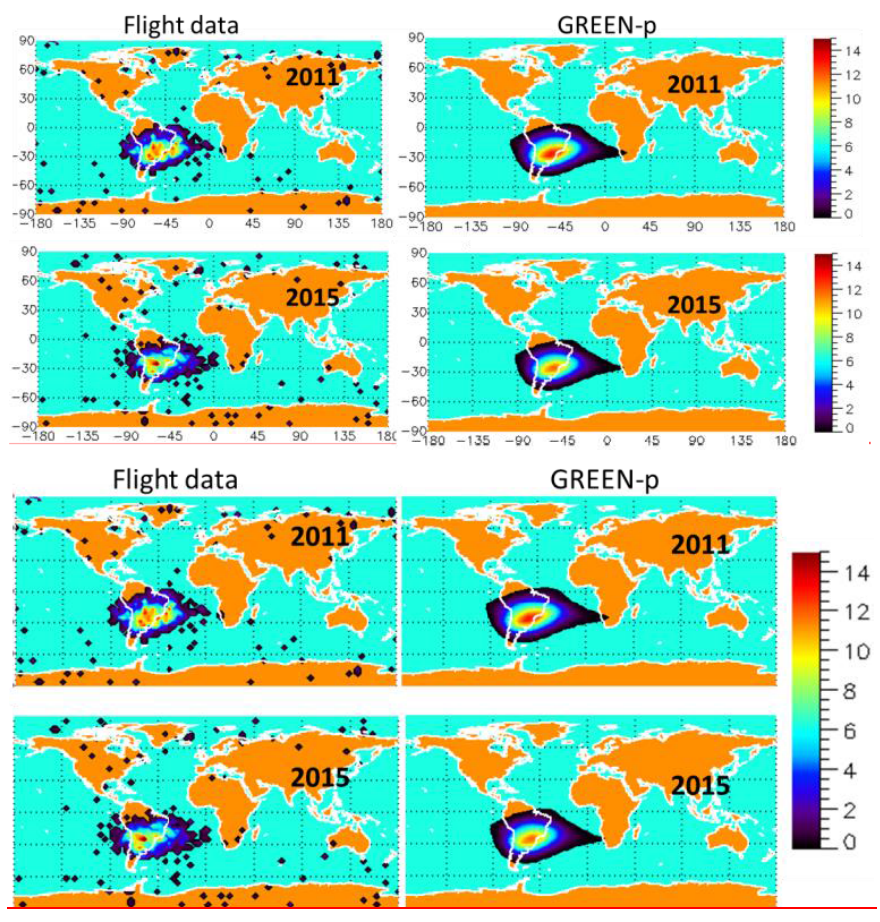

Fig. 12. SEU measured (left) onboard CryoSat-2/SIRAL payload and (right) predicted by GREEN-p model in a longitude-latitude map for two years (2011 early rising phase of the solar cycle and 2015 one year after solar maximum phase).

exhibit a general trend: predicted SEU rates are declining along years. This feature cannot be attributed to an orbital effect as CryoSat-2 orbit remains unchanged throughout the time period under study. Predictions from the GREEN-p model track quite nicely in-flight data from 2011 to 2019. Nevertheless, a more or less similar trend as in AP9 predictions 


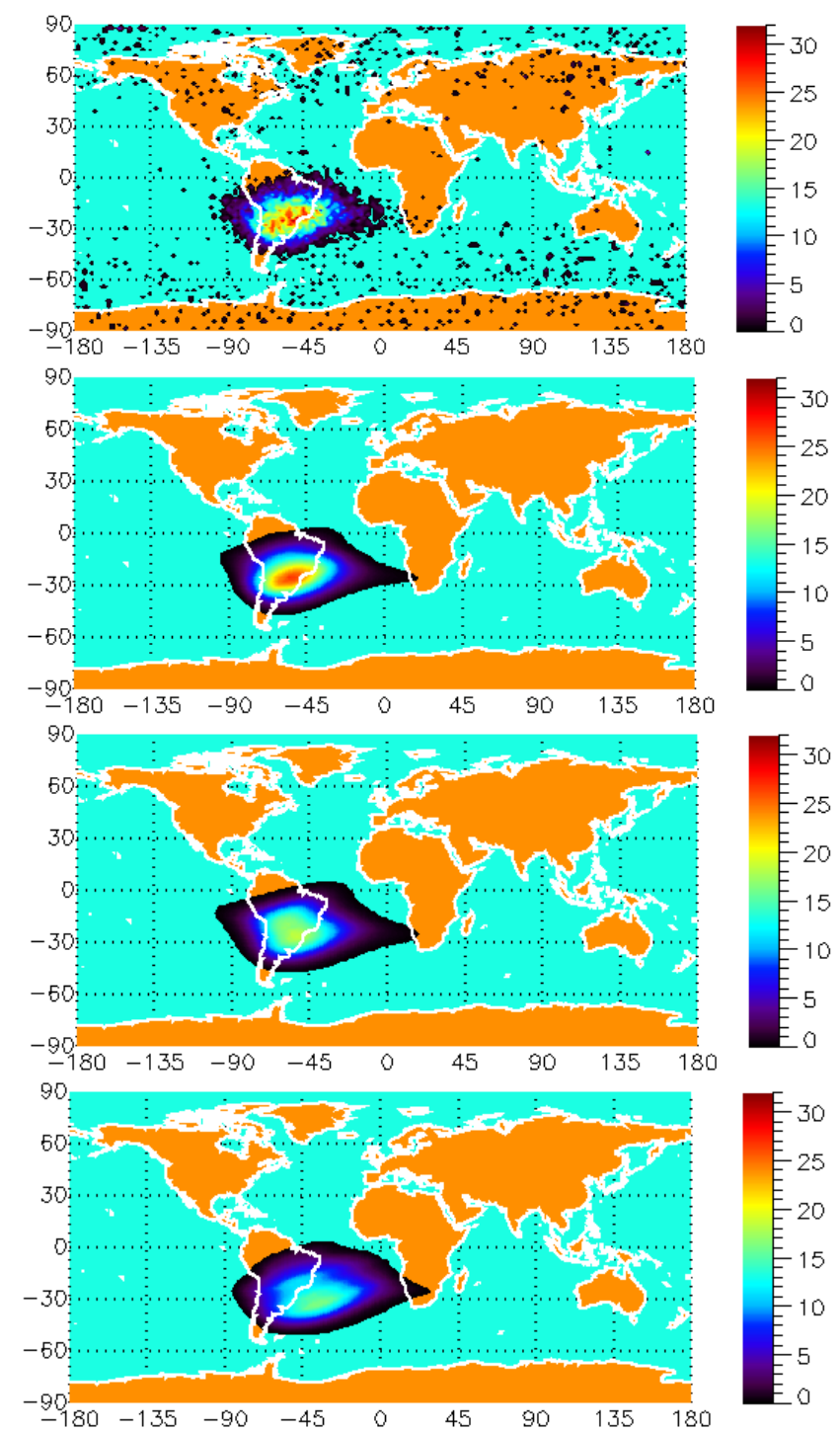

Fig. 13. SEU onboard the CryoSat-2/SIRAL payload from November 1, 2010, to March 31, 2019, in a longitude-latitude map, measured in situ (flight data on top panel), predicted from specification models using the Weibull fit "high" (second from top: GREEN-p, third from top: AP9 Mean V1.50.001, and bottom: AP8 $\mathrm{min}$ ).

is suspected. In 2011, GREEN-p overestimates the SEU daily rates a little bit, while it underestimates them in 2019. This feature is suspected to be related to International Geophysical Reference Field (IGRF)-12 which is being used in AP9 and in GREEN-p. IGRF-12 is extrapolated after 2015-2020 and may not represent accurately the real Earth's internal magnetic field. Definitive IGRF coefficients to be used between 2015 and 2020 will be made available only during year 2020 .

As shown before, SEU rate predictions obtained from AP8 solar min, AP8 solar max, AP9 Mean V1.50.001, and GREEN-p are closer to observations when the Weibull fit "high" is being used.

SEU measured onboard CryoSat-2/SIRAL payload and predicted by GREEN-p model is given in a longitude-latitude map for two years of the solar cycle in Fig. 12. The bin size in each plot is $4^{\circ}$ in longitude and latitude. In the flight data as well as in GREEN-p model prediction, it is found that the
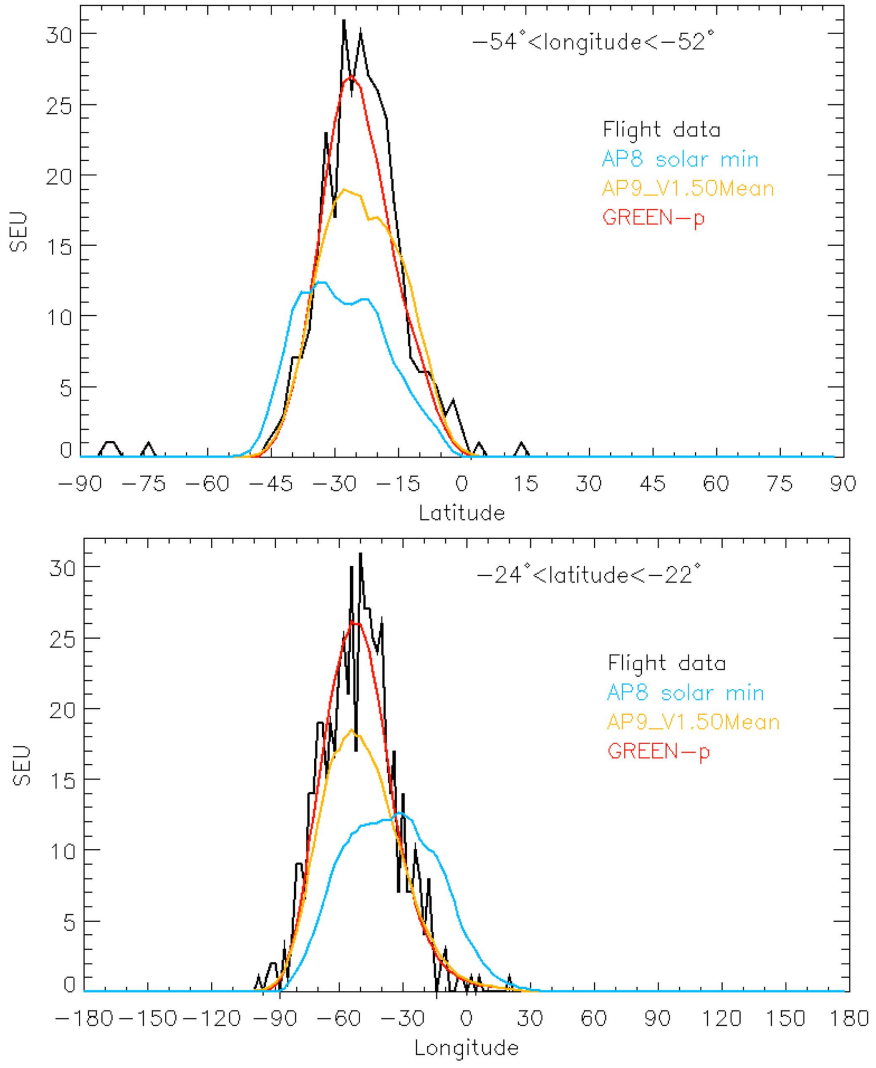

Fig. 14. (Top) SEU latitudinal profile at $-53^{\circ}$ longitude and (bottom) SEU longitudinal profile at $-23^{\circ}$ from flight data and specification model predictions.

heart of the SAA (where events are more numerous) is more extended in 2011 than in 2015, that is, the SAA shrink in solar maximum phase.

To validate specification models in deep details, predicted SEU along all the period under study is plotted in a longitude-latitude map and compared to flight data (Fig. 13). The bin size in each plot is $2^{\circ}$ in longitude and latitude. The color plot provides the total number of events recorded (or predicted) in a bin from November 1, 2010, to March 31, 2019. AP9 Mean V1.50.001 and GREEN-p locate the SAA at the right place, while AP8 min locates the SAA too far eastward and southward. This is not a surprise and these results are attributed to the magnetic field model being implemented in the specification models. AP9 and GREEN-p are defined with a magnetic field model depending on the year of interest, while AP8 min is forced to use a magnetic field model from the 1970s. The shape of the mapping in the SAA obtained also differs somehow from model to model. Note that the overall shape predicted by AP9 Mean and GREEN-p looks very similar to each other and to the flight data.

Nevertheless, they exhibit differences in the shape of the heart of the SAA: GREEN-p is predicting isocontour more oval than AP9 Mean. This feature is also found in the flight data. Finally, the peak SEU predicted by GREEN-p is the closest to flight data (underestimating by $14.7 \%$ ), while AP9 Mean V1.50.001 and AP8 min models underestimate the peak by $40.6 \%$ and $46.5 \%$, respectively. To better illustrate this 


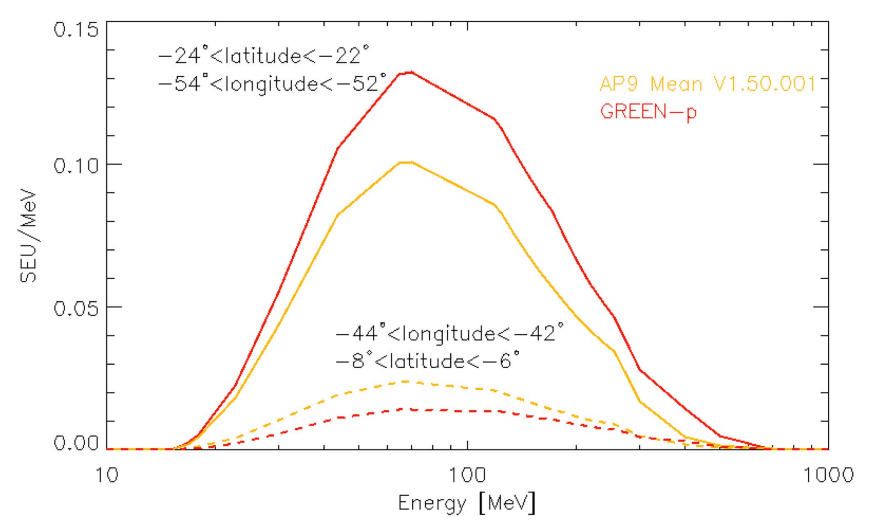

Fig. 15. Number of events per MeV predicted by AP9 Mean V1.50.001 and GREEN-p at two locations (one in the heart of the SAA: top two curves; and one at the outer edge of the SAA: bottom two curves).

result, a vertical $\left(-54^{\circ}<\right.$ longitude $\left.<-52^{\circ}\right)$ and horizontal cut $\left(-24^{\circ}<\right.$ latitude $\left.<-22^{\circ}\right)$ in the longitude-latitude maps from Fig. 13 is given in Fig. 14 where the three specification model outputs are compared to flight data. Note that AP8 min prediction is well below flight data but this discrepancy is mainly due to the wrong SAA location found with AP8 min and does not reflect a peak-to-peak discrepancy.

A detailed look at each energy contribution to the number of SEUs over the full time period is given at two locations, one in the heart of the SAA and one at the outer edge of the SAA in Fig. 15. It turns out that GREEN-p predicts a higher contribution at all energies in the heart of the SAA and a lower one at the outer edge of the SAA than AP9 Mean V1.50.001. This is consistent with results shown in Figs. 6 and 7.

\section{Discussion}

Solar cycle variations of trapped protons flux at low altitudes have been investigated by several authors [12]-[16]. A common feature was found: the long-term trapped proton flux exhibits a modulation along solar cycle larger than predicted by the models and out of phase with the solar radio flux F10.7. A lag time on the order of several hundred days (depending on the proton energy) is also pointed out. Moreover, years after years, the SAA is drifting westward.

This study concentrates on trapped proton environment with energies greater than $40 \mathrm{MeV}$ at $715-\mathrm{km}$ altitude along the synchronous orbit of Sun for eight years. The long-term prediction of SEU events recorded by an EDAC implemented on a TAS's payload flying onboard CryoSat-2 spacecraft are within $30 \%$ to flight data (except for AP8 max) when AP8 min, AP9 Mean V1.50.001, or GREEN-p are considered. Note that all three specification models have been developed independently and by different groups. So, having all three long-term predictions close one to the other suggest that they should be very much representative to the long-term (multiyears) true state. On shorter time scales (one year), a 30\% modulation along solar cycle 24 is found from CryoSat-2/SIRAL/EDAC data. Such a result reinforces previous results [12], [14], [16]. So far, among AP9 Mean V1.50.001, AP8 min, AP8 max, and GREEN-p, only GREEN-p model appears to be the most suitable proton specification model to reproduce the solar cycle modulation with a one-year time resolution seen in flight observations. Such a detailed knowledge of the space environment allows revisiting the margin policy that must be applied to design space missions. In particular, it allows considering margins attributed to specification model uncertainties well below the de facto factor 2 being often suggested at least at LEO. Accessing the solar cycle modulation of the space environment also allows to better access margins attributed to the environment for mission duration shorter than a solar cycle.

\section{ACKNOWLEDGMENT}

The authors would like to thank the CNES R\&T program for their full support to this activity.

\section{REFERENCES}

[1] D. M. Sawyer and J. I. Vette, "AP8 trapped proton model environment for solar maximum and minimum," Nat. Space Sci. Data Center, Greenbelt, MD, USA, Tech. Rep. NSSDC/WDC-A-R\&S 76-06, Dec. 1976.

[2] J. I. Vette, "The AE-8 trapped electron model environment," Nat. Space Sci. Data Center, Greenbelt, MD, USA, Tech. Rep. NSSDC/WDC-A-R\&S 91-24, 1991.

[3] G. P. Ginet et al., "AE9, AP9 and SPM: New models for specifying the trapped energetic particle and space plasma environment," in The Van Allen Probes Mission, N. Fox and J. L. Burch, Eds. Boston, MA, USA: Springer, Mar. 2013, pp. 579-615, doi: 10.1007/978-1-4899-7433-4_18.

[4] A. Sicard, D. Boscher, S. Bourdarie, D. Lazaro, D. Standarovski, and R. Ecoffet, "GREEN: The new global radiation earth environment model (beta version)," Ann. Geophys., vol. 36, no. 4, pp. 953-967, Jul. 2018, doi: 10.5194/angeo-36-953-2018.

[5] S. Bourdarie et al., "Benchmarking ionizing space environment models," IEEE Trans. Nucl. Sci., vol. 64, no. 8, pp. 2023-2030, Aug. 2017, doi: 10.1109/TNS.2017.2654687.

[6] FASTRAD. Accessed: Sep. 6, 2019. [Online]. Available: http://www. fastrad.net/

[7] C. Poivey, B. Doucin, M. Bruggemann, and R. Harboe-Sorensen, "Radiation characterisation of commercially available $1 \mathrm{Mbit} / 4 \mathrm{Mbit}$ SRAMs for space applications," in Proc. IEEE Radiat. Effects Data Workshop, Newport Beach, CA, USA, Jul. 1998, pp. 68-73.

[8] D. Novak et al., "In flight SEU tests on the European SMART-1 spacecraft," in Proc. 8th Eur. Conf. Radiat. Effects Compon. Syst., Cap d'Agde, France, Sep. 2005, pp. PH1-1-PH1-3, doi: 10.1109/RADECS. 2005.4365620.

[9] B. Vandevelde, "Proton test report: Single event effects M65608E (DC1203) Rad. Tolerant 128k × 8, 5-Volt SRAM From ATMEL," Test RADiation, Labège, France, Tech. Rep. TRAD/TP/M65608/ 1203/ESA/BV/1312, 2013.

[10] Celestrak. Accessed: Apr. 10, 2019. [Online]. Available: http://www. celestrak.com/NORAD/elements/

[11] Celestrak. Accessed: Apr. 10, 2019. [Online]. Available: http://celestrak. com/NORAD/documentation/spacetrk.pdf

[12] M. Qin, X. Zhang, B. Ni, H. Song, H. Zou, and Y. Sun, "Solar cycle variations of trapped proton flux in the inner radiation belt," J. Geophys. Res. Space Phys., vol. 119, pp. 9658-9669, Dec. 2014, doi: 10.1002/2014JA020300.

[13] J. Domingos, D. Jault, M. A. Pais, and M. Mandea, "The south Atlantic anomaly throughout the solar cycle," Earth Planet. Sci. Lett., vol. 473, pp. 154-163, Sep. 2017.

[14] C. I. Underwood, M. K. Oldfield, C. S. Dyer, and A. J. Sims, "Long term trend in the LEO radiation environment as measured by the radiation monitors on-board three UoSAT-class micro-satellites," in Proc. Environ. Modeling Space-Based Appl., Symp. (ESA SP-392), W. Burke and T.-D. Guyenne, Eds. Noordwijk, The Netherlands: ESTEC, Sep. 1996, p. 37.

[15] F. F. Badavi, S. A. Walker, and L. M. S. Koos, "Low earth orbit assessment of proton anisotropy using AP8 and AP9 trapped proton models," Life Sci. Space Res., vol. 5, pp. 21-30, Apr. 2015.

[16] W. Miyake, Y. Miyoshi, and A. Matsuoka, "An empirical modeling of spatial distribution of trapped protons from solar cell degradation of the Akebono satellite," Adv. Space Res., vol. 56, pp. 2575-2581, Dec. 2015. 